\title{
High proportions of bacteria are culturable across major biomes
}

\author{
Adam C. Martiny $\mathbb{1}^{1,2}$
}

Received: 19 August 2018 / Revised: 11 January 2019 / Accepted: 28 February 2019 / Published online: 5 April 2019

(c) International Society for Microbial Ecology 2019

\begin{abstract}
The paradigm that only $1 \%$ of microbes are culturable has had a profound impact on our understanding of microbial ecology and is still a major motivation for mostly using molecular tools to characterize microbial communities. However, this point is often expressed vaguely, suggesting that some scientists have different interpretations of the paradigm. In addition, there have been substantial advances in cultivation techniques suggesting that this paradigm may no longer be correct. To quantify bacterial culturability across six major biomes, I found that the median 16S rRNA similarity of bacteria to known cultured relatives was $97.3 \pm 2.3 \%$ (s.d.). Furthermore, $52.0 \pm 24 \%$ of sequences and $34.9 \pm 23 \%$ of taxa (defined as $>97 \%$ similar) had a closely related cultured relative. Thus, many cells and taxa across environments are culturable with known techniques, suggesting that the $1 \%$ paradigm is no longer correct.
\end{abstract}

Historically, the diversity and physiology of microorganisms were assessed using cultivation techniques [1], but $16 \mathrm{~S}$ rRNA sequencing revealed high unrecognized diversity leading to the current paradigm that only $1 \%$ of microorganisms are culturable. The paradigm had a profound impact on the field of microbiology and is continually used as motivation for employing — and developing new—molecular techniques for characterizing microbial communities.

There seem to be three interpretations for the ' $1 \%$ culturability paradigm' in the literature. The first interpretation (H1) is that $1 \%$ of cells in a community can be cultivated. This interpretation suggests that, even if all currently available methods are applied, one could only culture $1 \%$ of bacterial cells from a sample. A second interpretation $(\mathrm{H} 2)$ is that only $1 \%$ of taxa in a community can be cultivatedagain using all available methods. Here, a taxon is defined as a group of closely related organisms (e.g., using a sequence similarity criterion). The difference between the first and

Supplementary information The online version of this article (https:// doi.org/10.1038/s41396-019-0410-3) contains supplementary material, which is available to authorized users.

Adam C. Martiny

amartiny@uci.edu

1 Department of Earth System Science, University of California, Irvine, CA 92697, USA

2 Department of Ecology and Evolutionary Biology, University of California, Irvine, CA 92697, USA second interpretation is that the second gives equal weight to abundant vs. rare taxa. The third interpretation (H3) is more operational and posits that $1 \%$ of cells in a community grow when plated on a standard agar medium. The third version is based on the observation that plate-counting, in comparison to direct staining techniques, grossly underestimate bacterial abundances and diversity [2].

The origin of the ' $1 \%$ culturability paradigm' is rather murky. Many papers refer back to reviews by Torsvik and Øvreås [3] or Amann and co-workers [4]. However, the former does not provide a reference or an analysis to support the paradigm. Amann and co-workers present a table comparing agar plate- vs. direct-counts so $\mathrm{H} 3$ is likely the original interpretation. In contrast, a Google Scholar search reveals that current usage of the ' $1 \%$ culturability paradigm' seems to more often refers to $\mathrm{H} 1$ or H2 (Fig. S1), although the point is often vaguely expressed and can be difficult to categorize. For several decades, there has been a concerted effort to cultivate abundant microorganisms across environments, and many methodological improvements have been developed [5-7]. Historically, there was a low proportion of abundant lineages in culture across most biomes, but I hypothesize that these recent cultivation innovations and increased efforts have resulted in much higher proportions of culturable bacteria today. Hence, I expect that much more than $1 \%$ of both cells $(\mathrm{H} 1)$ or taxa $(\mathrm{H} 2)$ from a variety of environments have been cultivated with existing techniques.

Here, the proportion of microorganisms with known culturable relatives was quantified from 40 communities 

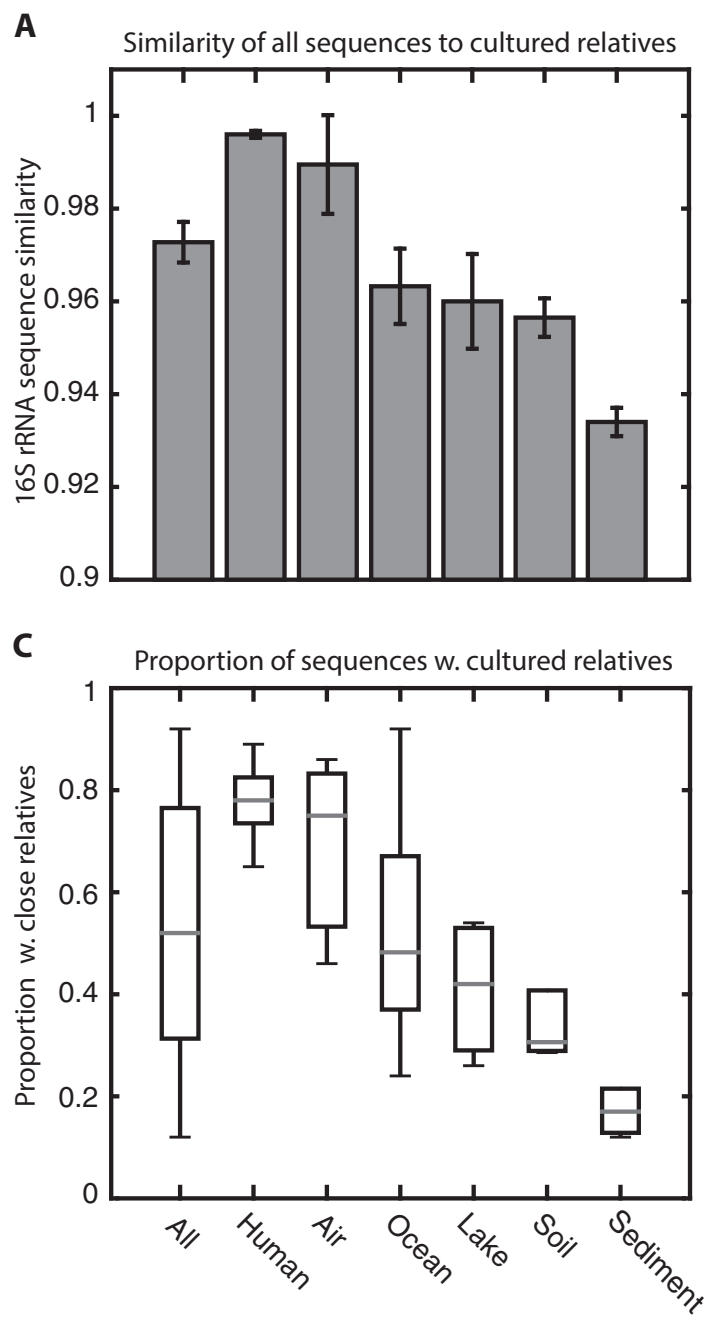

Fig. 1 16S rRNA sequence similarity between community members and known isolates. a The median similarity between amplicon sequences and known isolates as a test of the ' $1 \%$ culturability paradigm', H1. b The median similarity between representative amplicon sequences and known isolates as a test of $\mathrm{H} 2$. The error bars represent one standard error. c Box plot of proportion of sequences with a close relative in culture across biomes. d Proportion taxa with a close

across six major biomes (human associated, marine water, marine sediments, freshwater, soil, and air, Table S1). The analysis was restricted to Sanger sequences as these are longer and commonly have a lower error rate due to overlapping sequence runs. One hundred sequences were randomly retrieved from each sample and compared using 'SeqMatch' against known cultured microorganisms classified in RDP [8].

To test the $1 \%$ of cells version of the paradigm (H1), the similarity of directly amplified 16S rRNA sequences was compared to known isolates (Fig. S2). The median similarity between amplicon sequences and isolates across all biomes was $97.3 \pm 2.3 \%$ (s.d.) but with significant variation across biome type (1-way ANOVA, $p<0.0001$, Fig. 1a). The median similarity to isolates in human-associated
B Similarity of all taxa to cultured relatives

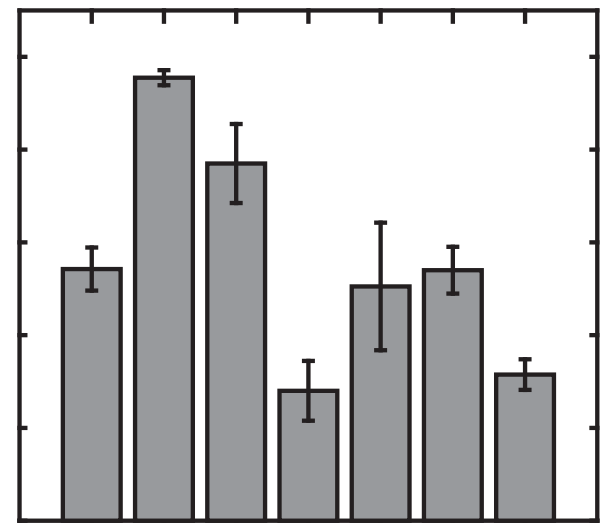

D Proportion of taxa w. cultured relatives

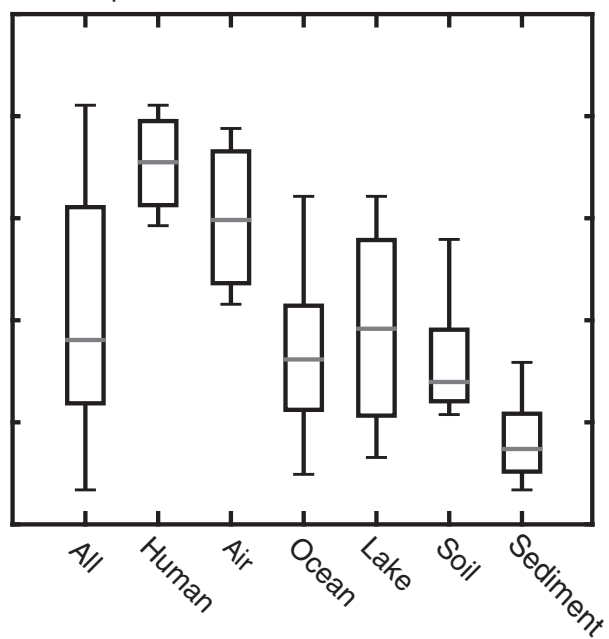

relative in culture as distributed across biomes. A taxon as well as a close cultured relative are here defined as 16S rRNA sequences sharing $>97 \%$ similarity and binned using MOTHUR using the opticlust algorithm [9]. For the box plots, the gray line is the median, the box is the 25-75 percentile, and the errorbar is the 2.5-97.5 percentile of observations. See Table S1 for sample list

communities was $>99 \%$, but only $~ 93 \%$ in marine sediments (Fig. 1a). If we initially define 'closely-related' as sequences sharing at least $97 \%$ 16S rRNA similarity, on average $52.0 \pm 24 \%$ of sequences had a closely-related known isolate but the proportion varied significantly across biomes (1-way ANOVA, $p<0.0001$, Fig. 1c). The high median similarity between directly retrieved sequences and known isolates as well as high proportions of sequences with a closely related cultured relative indicate that there is limited support today for $\mathrm{H} 1$ of the ' $1 \%$ culturability paradigm'.

The $1 \%$ of taxa interpretation $(\mathrm{H} 2)$ was tested by comparing the similarity between a representative of each taxon (grouped at 97\% 16S rRNA similarity using MOTHUR [9]) and known isolates (Fig. S3). Here, the median similarity 


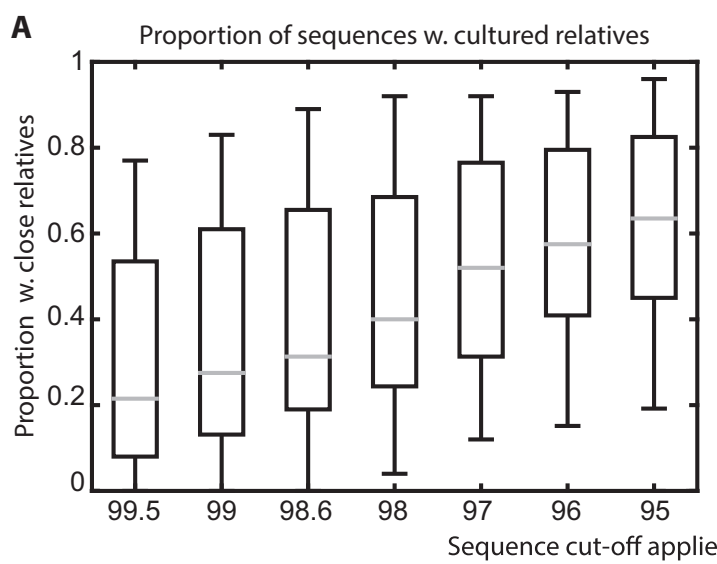

B Proportion of taxa w. cultured relatives

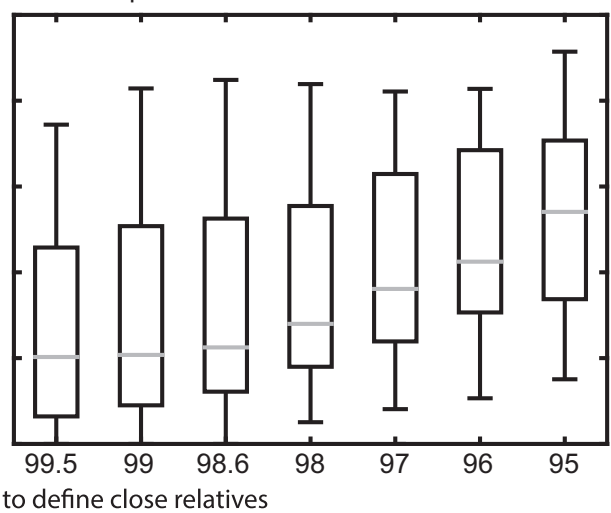

Fig. 2 Similarity between community members and known isolates as a function sequence threshold to define 'closely-related'. a Proportion of sequences (H1) with cultured relatives across definitions of 'closelyrelated'. b Proportion taxa $(\mathrm{H} 2)$ with cultured relatives across

between taxa and known isolates was $95.4 \pm 2.9 \%$ but again with a significant difference across biomes (one-way ANOVA, $p<0.0001$, Fig. 1b). Taxa from human associated communities shared the highest, and sediments the lowest, similarity to known isolates. It is notable that the proportions of sequences vs. taxa with cultured relatives did not always follow the same trend. For ocean communities, more than $50 \%$ of sequences but only $35 \%$ of taxa had known cultured relatives whereas little difference was seen in other biomes-perhaps a result of the coordinated effort to isolate abundant marine lineages [5]. On average $34.9 \%$ of taxa across biomes were at least $97 \%$ related to known isolates and the proportion significantly varied between biomes (one-way ANOVA, $p<0.0001$, Fig. 1d). Part of the difference in culture representation among biomes was driven by the alpha diversity profile, whereby more diverse communities (e.g., sediments) contained a lower proportion of cultured representatives (Fig. S4). Nevertheless, the proportion of sequences $(\mathrm{H} 1)$ and taxa $(\mathrm{H} 2)$ represented in culture was much higher than $1 \%$, revealing limited support for either versions 1 or 2 of the ' $1 \%$ culturability paradigm'.

I next wanted to test if the proportion of microorganisms with a known relative is affected by the sequence library size. Indeed, the observed proportion of taxa-but not sequences-with closely related cultured relatives are dependent on the size of the sequencing library sample (Fig. S5). Thus, the extent of inclusion of 'the rare biosphere' affects $\mathrm{H} 2$ but not $\mathrm{H} 1$ and a sample library of $>1$ million short sequences will likely yield a low proportion of cultured taxa.

The proportion of sequences or taxa with a known relative is expected to scale with our definition of sequence similarity threshold determining if something is the same vs. different. The $97 \%$ sequence similarity threshold is commonly applied in microbial ecology, but a more stringent threshold yielded definitions of 'closely-related'. For the box-plot, the gray line is the median, the box is the $25-75$ percentile, and the errorbar is the 2.5-97.5 percentile of observations $(n=40)$. Here, taxa and closelyrelated are both defined using a range of sequence similarities

lower proportions of cultured relatives in the samples and vice-versa (Fig. 2). It is important to recognize that $16 \mathrm{~S}$ rRNA similarity is non-linearly related to genome similarity [10]. Thus, the implications of this finding for understanding culturability of microorganisms is complex as closely related organisms have some shared but also some unique traits. However, a more stringent threshold is sensitive to efforts in culturing, sequencing, and reporting isolates within wellestablished (and culturable) groups as this would result in better subclade representation. In conclusion, the definition of the diversity unit is important for how we should interpret the paradigm.

Accepting or rejecting the ' $1 \%$ culturability' paradigm for bacteria depends on key details in how the paradigm is interpreted, how the diversity unit is defined, and the biome of interest. Three versions of the paradigm show varying degrees of support. The first-1\% of cells-and most common version of the paradigm is most clearly rejected as the median similarity between cells, and existing cultures is above $97 \%$ across a range of biomes. The acceptance or rejection of the second-1\% of taxa-version is more ambiguous. Thirty-four percent of taxa (defined at $97 \%$ sequence similarity) across biomes have a closely related relative in culture, but the proportions vary greatly across samples. The degree of the culture representation among taxa is also sensitive to the library size and the associated presence of less common members. The third interpretation was not analyzed in detail as few would dispute that cultivation on standard agar plates grossly underestimate the diversity and abundance of microorganisms (with a few notable exceptions [6]). Part of the variability in culture representation is associated with the diversity of the sample and potential for missing rare members, but overall culturing effort and knowledge of nutritional requirement likely play a role, too $[5,11]$. There was a time when the ' $1 \%$ 
culturability paradigm' across all interpretations applied, and many (or most lineages) were not in culture. However, the extensive effort on culturing especially abundant lineages has led to an observed proportion that is much higher than $1 \%$ when using a conventional taxon definition. Thus, it is time to update the ' $1 \%$ culturability paradigm' and recognize that many if not most abundant lineages across diverse biomes are already in culture.

Acknowledgements I would like to thank Alyse Larkin, Sydney Glassman, and Jennifer Martiny for many helpful comments and acknowledge support for this work from NSF (OCE-1559002, OCE-1848576, and DEB-1457160) and the U.S. Department of Energy, Office of Science, Office of Biological and Environmental Research (BER), under award number (DE-SC0016410).

\section{Compliance with ethical standards}

Conflict of interest The author declare that he has no conflict of interest.

Publisher's note: Springer Nature remains neutral with regard to jurisdictional claims in published maps and institutional affiliations.

\section{References}

1. Brock TD. The study of microorganisms in situ: progress and problems. Symp Soc Gen Microbiol. 1987;41:1-17.
2. Staley JT, Konopka A. Measurement of in situ activities of nonphotosynthetic microorganisms in aquatic and terrestrial habitats. Annu Rev Microbiol. 1985;39:321-46.

3. Torsvik V, Øvreås L. Microbial diversity and function in soil: from genes to ecosystems. Curr Opin Microbiol. 2002;5:240-5.

4. Amann RI, Ludwig W, Schleifer K-H. Phylogenetic identification and in situ detection of individual microbial cells without cultivation. Microbiol Rev. 1995;59:143-69.

5. Giovannoni S, Stingl U. The importance of culturing bacterioplankton in the 'omics' age. Nat Rev Microbiol. 2007; 5:820-6.

6. Joseph SJ, Hugenholtz P, Sangwan P, Osborne CA, Janssen PH. Laboratory cultivation of widespread and previously uncultured soil bacteria. Appl Environ Microbiol. 2003;69:7210-5.

7. Kaeberlein T, Lewis K, Epstein SS. Isolating 'uncultivable' microorganisms in pure culture in a simulated natural environment. Science. 2002;296:1127-9.

8. Cole JR, Wang Q, Fish JA, Chai B, McGarrell DM, Sun Y, et al. Ribosomal Database Project: data and tools for high throughput rRNA analysis. Nucleic Acids Res. 2014;42. https://doi.org/10. 1093/nar/gkt1244.

9. Schloss PD, Westcott SL, Ryabin T, Hall JR, Hartmann M, Hollister EB, et al. Introducing mothur: open-source, platformindependent, community-supported software for describing and comparing microbial communities. Appl Environ Microbiol. 2009;75:7537-41.

10. Rosselló-Móra R, Amann R. Past and future species definitions for Bacteria and Archaea. Syst. Appl. Microbiol. 2015;38:209-216.

11. Van Der Star WRL, Miclea AI, Van Dongen UGJM, Muyzer G, Picioreanu C, Van Loosdrecht MCM. The membrane bioreactor: a novel tool to grow anammox bacteria as free cells. Biotechnol Bioeng. 2008;101:286-94. 